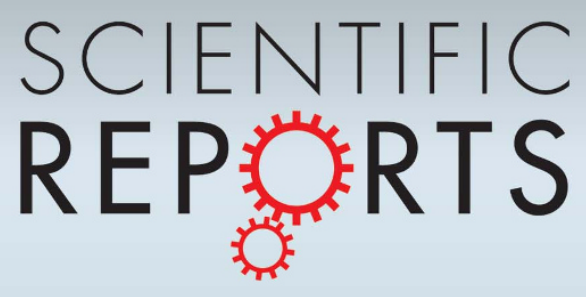

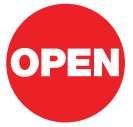

SUBJECT AREAS:

BIOCHEMISTRY

MEMBRANE DYNAMICS

MOLECULAR ENGINEERING

BIOPHYSICS

Received

3 May 2011

Accepted

25 October 2011

Published

28 November 2011

Correspondence and requests for materials should be addressed to S.Z. (Shuguang@MIT.

EDU)

\section{Structure and function analyses of the purified GPCR human vomeronasal} type 1 receptor 1

\author{
Karolina Corin', Philipp Baaske², Sandra Geissler², Christoph J. Wienken³, Stefan Duhr², Dieter Braun ${ }^{3}$ \\ \& Shuguang Zhang'
}

${ }^{1}$ Center for Biomedical Engineering, NE47-379, Massachusetts Institute of Technology, 77 Massachusetts Avenue, Cambridge, MA 02139-4307, USA, ${ }^{2}$ NanoTemper Technologies GmbH, Amalienstrasse 54, 80799 München, Germany, ${ }^{3}$ Systems Biophysics, Functional Nanosystems, Department of Physics, Ludwig-Maximilians University München, Amalienstrasse 54, 80799 München, Germany.

The vomeronasal system is one of several fine-tuned scent-detecting signaling systems in mammals. However, despite significant efforts, how these receptors detect scent remains an enigma. One reason is the lack of sufficient purified receptors to perform detailed biochemical, biophysical and structural analyses. Here we report the ability to express and purify milligrams of purified, functional human vomeronasal receptor hVN1R1. Circular dichroism showed that purified hVN1R1 had an alpha-helical structure, similar to that of other GPCRs. Microscale thermophoresis showed that hVN1R1 bound its known ligand myrtenal with an $\mathrm{EC}_{50} \sim 1 \mu \mathrm{M}$. This expression system can enable structural and functional analyses towards understanding how mammalian scent detection works.

A

lthough the existence and function of a human vomeronasal organ (VNO) is controversial, almost all studies agree that a vomeronasal organ is present in the developing fetus. However, many studies report that this organ is vestigial, and function is lost in adults, while others report development of a mature organ ${ }^{1-6}$. Interestingly, one study found that the ability to detect the adult vomeronasal organ varies; they could not find vomeronasal organs in subjects that had clearly identifiable vomeronasal organs on prior inspections, and vice versa ${ }^{1}$.

In most mammals, signals from the vomeronasal organ are directed to the accessory olfactory bulb (AOB). However, studies in humans suggest that degenerative changes occur in the fetal accessory olfactory bulb, and that it isn't present in adults ${ }^{4,5}$. Most studies that used neuron-specific stains failed to find evidence of nerve bundles ${ }^{1,6}$. Others suggest the presence of neurons in the adult vomeronasal organ, albeit at a low density ${ }^{7}$. In spite of this, some studies suggest pheromonal responses in humans directly through the vomeronasal organ. Androstadienone applied directly to the female vomeronasal organ decreased nervousness, tension, and negative feeling states. Decreases in cardiac frequency and respiratory frequency, and increases in body temperature and alpha-coritcal activity were also measured ${ }^{8}$. When a steroidal vomeropherin was applied to the male vomeronasal organ, a decrease in follicle-stimulating hormone and luteinizing hormone pulsatility was observed. Changes in cardiac frequency, respiratory frequency, and other physiological responses were also observed ${ }^{9}$.

Taken together, current evidence suggests that humans may have a potentially functional vomeronasal organ that functions differently than in other mammals. However, until recently, it was believed that human vomeronasal receptors (VNRs) were all pseudogenes. In 2002, Rodriguez and Mombaerts found 5 VNR type 1 (VN1R) genes with open reading frames. They were successfully expressed in $\mathrm{HeLa} / \mathrm{Olf}$ cells ${ }^{11}$. Interestingly, all five were found to respond to volatile molecules and signal through the canonical olfactory signaling pathway. Even more curiously, mRNA of one receptor - hVN1R1 - was detected in several other tissues, including the main olfactory epithelium (MOE), brain, lung, and kidney ${ }^{12}$. This raises the possibility that human VN1Rs may function more like olfactory receptors in the MOE, or may have non-olfactory functions.

Studying human vomeronasal receptors at the molecular level may shed light on both their function and that of the human vomeronasal organ. However, the difficulty of functionally expressing and purifying these G-protein coupled receptors in sufficient quantities is a major obstacle towards research. Only one report has produced functional receptors in cells, and identified potential ligands ${ }^{11}$. However, in order to study their structure and function, it is necessary to produce and purify milligram-scale quantities. Here, we report a method of expressing 
and purifying milligram-level quantities of the human receptor hVN1R1 from HEK293 cells. Circular dichroism indicated that the purified receptor was properly folded with stable $\alpha$-helical domains. Microscale thermophoresis assays showed that the purified receptor was able to bind to its ligand. Our expression platform provides the basis for carrying out further structural analyses of the human vomeronasal receptors, and can enable screens for crystallization trials. It could not only aid in future studies of human VNRs and help identify their biological role, it could also provide a basis for designing biologically inspired devices like artificial noses.

\section{Results}

Induction of hVN1R1 expression in stable HEK293 cell lines. Producing milligram quantities of homogenous membrane protein samples is notoriously difficult. Over-expression of these proteins in mammalian cells is often reported to have toxic effects, which reduce the total protein yield. Additionally, constitutive expression of such proteins yields non-homogeneous samples, as proteins can be in various stages of synthesis, degradation, or post-translational modification. To minimize toxic effects, and to generate a homogenous protein sample, the Invitrogen T-REx system was used to create stable hVN1R1- inducible cell lines ${ }^{13}$. This system allows large batches of cells to be grown and simultaneously induced to express protein.

Sodium butyrate has previously been used to enhance tetracyclineregulated protein expression ${ }^{13,14}$. Induction of VN1R1 expression was thus tested with tetracycline alone, and tetracycline combined with sodium butyrate at various concentrations. No detectable protein expression was seen in the absence of induction agents. Sodium butyrate combined with tetracycline enhanced protein expression 4-5 fold over induction with tetracycline alone. Significant cell death was observed in cultures treated with both tetracycline and sodium butyrate, indicating the toxic effects of protein expression. The clone with the highest expression, the least toxicity, and least degradation products was selected and expanded. For all subsequent experiments, the following induction condition was used: $1 \mu \mathrm{g} / \mathrm{ml}$ of tetracycline and $2.5 \mathrm{mM}$ of sodium butyrate for 48 hours.

SDS-PAGE and Western blotting analysis were used to characterize the protein samples. Immunoblotting against the rho1D4 tag revealed two bands, which correspond in size to monomeric and dimeric forms of the receptor. This size pattern has been reported for several olfactory receptors ${ }^{13,15,16}$, and is the first such reporting for a human vomeronasal receptor. The hVN1R1 sample also showed evidence of a degradation product.

Immunohistochemical staining of induced cells. Numerous studies report the difficulty of expressing GPCRs in heterologous systems, and particularly of targeting proteins to the membrane $e^{17-20}$. Glycosylation, particularly of specific conserved N-terminal sites, may be necessary for proper protein folding and localization ${ }^{21}$. However, glycosylation can lead to inhomogeneous samples and make crystal packing difficult. Here, we used the rho1D4-tagged protein with potential glycosylation sites removed. To determine whether removal of predicted glycosylation sites affected protein localization, induced cells were stained with antibodies against the rho1D4 tag. (Figure 1) Non-induced cells showed negligible fluorescence. Permeabilized cells stained with the rholD4 tag demonstrated membrane-localization of the protein. These results indicate that the protein is trafficked to the cell membrane. They further demonstrate that the hVN1R1 glycosylation sites are not necessary for appropriate protein localization in HEK293 cells, and indicate that they are not necessary for appropriate protein folding.

Systematic detergent screening for receptor solubilization. Selection of an appropriate detergent is critical for the successful

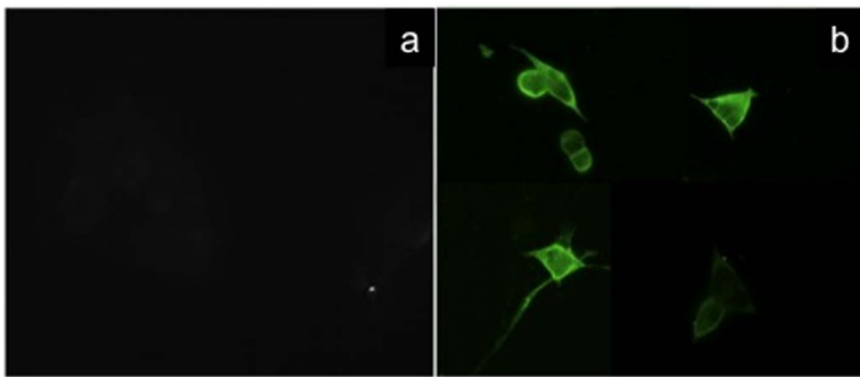

Figure $1 \mid$ Immunohistochemical staining of hVN1R1. (a) Non-induced HEK293 cells showed no staining. (b) Induced HEK293 cells stained with the rho1D4 antibody showed that the expressed hVN1R1 was localized to the cell membrane.

solubilization of a GPCR from a cell membrane, and for the subsequent purification and stabilization of the receptor. Since the optimal detergent can vary even between proteins in the same family, it must be empirically determined. Thus, we carried out a systematic detergent screen (Figure 2).

Ninety-six detergents were screened. These detergents belonged to one of four general classes: anionic, cationic, non-ionic, and zwitterionic. The detergents were chosen from a commercial Solution Master Detergent $\mathrm{Kit}^{22}$, and included detergents that have been successfully used to purify, solubilize, or crystallize GPCRs. Additionally, several detergent mixtures were tested as previously described $^{22}$. All detergents were used at a concentration of $2 \%$, which was above the critical micelle concentration, with the exception of MEGA-8.

The most effective detergents for hVN1R1 solubilization were the zwitterionic fos-choline series (\#71-78) and n-Dodecyl B-iminodpropionic acid (DIPA, \#89). The zwitterionic cyclofos7, cationic hexadecyl- and tetradecyltrimethylammonium chloride detergents $(\# 8,9)$, and anionic sodium dodecanoyl sarcosine (\#5) were also reasonably effective, while most other detergents failed to solubilize a significant amount of receptor. Similar results with other GPCRs have previously been reported ${ }^{22,23}$. The effectiveness of the fos-choline (FC) series to solubilize membrane proteins is not surprising: they are structurally related to phosphatidylcholine, a constituent of the phospholipid bilayer. Although FC15, FC16, and DIPA were able to solubilize more protein, FC14 was chosen for all subsequent experiments. The CMC for DIPA has not been established, and FC15 and FC16

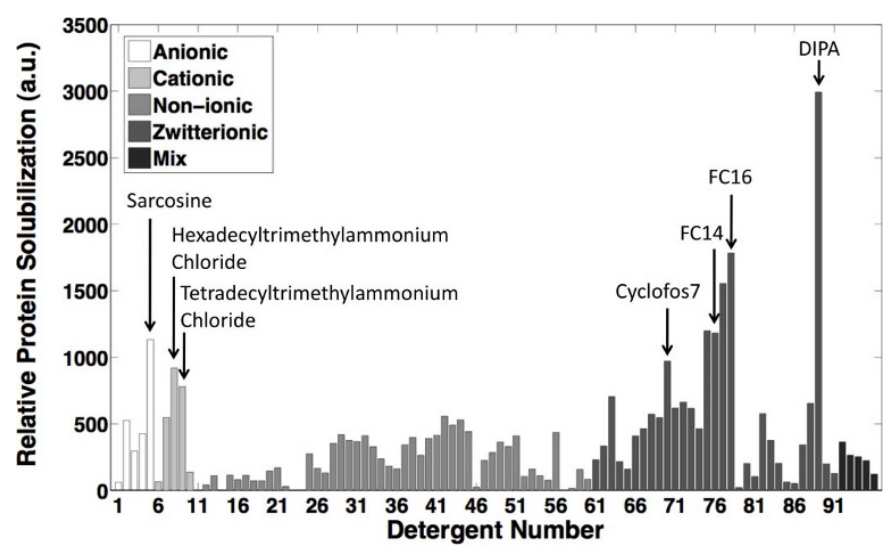

Figure $2 \mid$ Detergent screens for solubilizing and stabilizing hVN1R1. Zwitterionic detergents typically solubilized a greater portion of the expressed protein, though several cationic and anionic detergents were able to solubilize significant amounts. The fos-choline detergents were the subclass of detergents that solubilized the greatest amount of protein. 
have much lower CMCs, which could inhibit detergent substitution in downstream experiments. Furthermore, previous experiments indicate that the fos-choline family may promote proper folding as well as stabilize the protein structure ${ }^{24}$. Additionally, FC14 has previously been used for membrane protein solubilization and purification ${ }^{13,22,23,25}$, and has been used to crystallize and solve two high-resolution protein structures ${ }^{26,27}$.

Purification of HEK293-expressed hVN1R1. A two-step process using immunoaffinity chromatography and size-exclusion chromatography (SEC) was used to purify the expressed VN1R1 receptors (Figure 3$)^{13,23}$.

A small-scale purification was performed to determine the potential of using heterologous expression for large-scale protein production. Solubilized protein was first bound to rho1D4-tagged Sepharose 4B beads. After thoroughly washing the column to remove impurities, the protein was eluted with an excess of the rholD4 epitope peptide AcTETSQVAPA-NH 2 . To further purify the protein, remove the elution peptide, and separate the monomeric and dimeric forms, the receptor sample was subjected to SEC. The column flow through was monitored with UV absorption at $280 \mathrm{~nm}$ and $215 \mathrm{~nm}$, and was automatically collected in separate fractions. The UV spectrum showed 4 distinct peaks. The fractions corresponding to these peaks were pooled, concentrated, and analyzed by western blotting and silver staining. The first two peaks primarily corresponded to dimerized and aggregated protein, though the monomeric form was present throughout. The third peak corresponded to the monomeric form, which could be obtained at $>90 \%$ purity. The fourth peak was the elution peptide (Figure 3). Western blotting and total protein stains demonstrated that all of the expressed receptors had been captured by the beads. One gram of cells yielded approximately $1 \mathrm{mg}$ of total protein. Of the protein recovered after SEC, over $60 \%$ was the monomer. These yields are sufficient to obtain large quantities of highly pure receptor for biochemical analyses and crystal screens. Bioreactor suspension cultures could also potentially be used to increase the protein yield ${ }^{23}$.

Structural characterization of purified hVN1R1. Circular dichroism (CD) was used to determine whether the FC14-solubilized

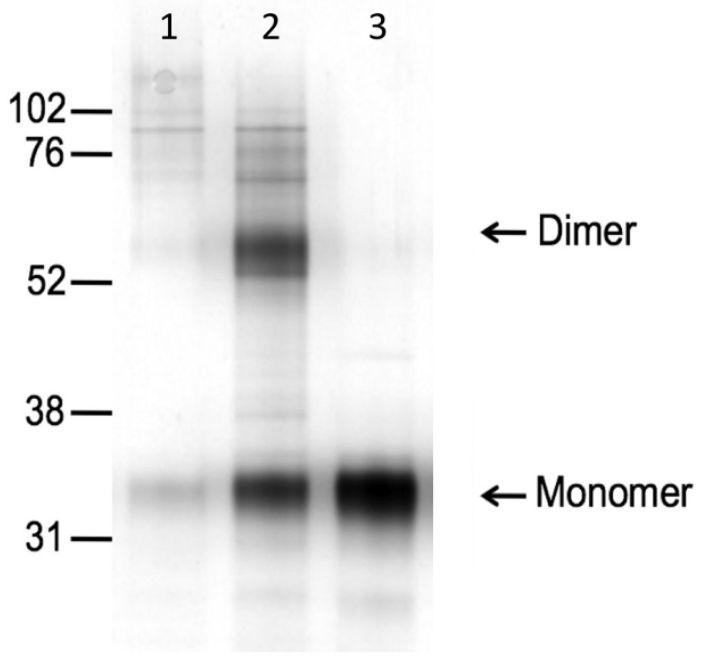

Figure 3 Silver stain of hVN1R1. Four distinct peaks were observed on the $280 \mathrm{~nm}$ UV trace through a size exclusion column (SEC). Samples corresponding to the peaks were pooled and run on a gel. The first peak (lane 1) primarily corresponded to higher molecular weight aggregates. The second peak (lane 2) contained dimerized receptor. The third peak (lane 3) contained a monomeric version of hVN1R1. This form of the receptor constituted over $60 \%$ of the protein recovered after SEC, and was present in all of the samples. The fourth peak contained the elution peptide, and is not shown. and purified hVN1R1 was properly folded (Figure 4). Far and near UV were used to probe the secondary and tertiary structure of the purified receptor. Figure $4 \mathrm{~A}$ shows the far UV spectrum. The CD spectrum showed a characteristic $\alpha$-helical shape with valleys at about 208 and $220 \mathrm{~nm}$. This is expected, since hVN1R1 is a GPCR with 7 predicted transmembrane helices. Figure $4 \mathrm{~B}$ shows the near UV spectrum. The peaks in the near UV spectrum indicate that the purified VN1R1 has a well-defined tertiary structure ${ }^{23,28}$. Together, the CD spectra suggest that HEK293-expressed hVN1R1 is properly folded, and that FC14 is able to maintain this structure once the receptor has been extracted from the cell membrane and purified.

Ligand binding assay of purified hVN1R1. Microscale thermophoresis (MST) was used to determine whether the FC14 solubilized receptor could bind its ligand myrtenal (MW 152.23). MST is the directed movement of molecules along a spatial temperature gradient. This movement is sensitive to changes in the hydration shell surrounding the molecule. Ligand-binding alters this shell in a way that measurably changes the molecules' thermophoretic movement ${ }^{29-31}$. MST yields results that are comparable to SPR and other binding assays ${ }^{32}$. However, unlike SPR or other surface-based techniques, MST does not require immobilization. The molecules are monitored in free solution. Additionally, proteins can be tracked by detecting the fluorescence of native tryptophans. Coupling-chemistries or other modifications that could potentially alter a receptors' function are thus not necessary.

The purified monomeric form of hVN1R1 was used for ligandbinding measurements. Samples were titrated with varying concentrations of myrtenal. Myrtenal has been reported as a ligand with an expected EC50 of $\sim 22 \mu \mathrm{M}$ in HeLa/Olf cells ${ }^{11}$. Figure 5 shows the MST titration curves normalized to the fraction of bound receptor. The monomer exhibits a plateau at low concentrations and at high concentrations. Fitting the Hill equation to the data yields a $\mathrm{K}_{\mathrm{D}}$ of $\sim 1 \mu \mathrm{M}$. This result is similar to the result reported by Shirokova et al, and is also in the same range as $\mathrm{K}_{\mathrm{D}}$ values measured for olfactory receptors $^{23,33}$. Boiled controls under otherwise identical conditions did not exhibit any plateaus, demonstrating that the measured changes in thermophoretic amplitude resulted from myrtenal binding to hVN1R1. Future studies must be performed to determine the effect dimerization has on hVN1R1 function.

\section{Discussion}

Our study shows that HEK293 cells can be used to produce milligram quantities of a human vomeronasal receptor. The purified receptors had alpha-helical secondary structures, suggesting that they were properly folded. They were also able to bind their reported small molecular ligand, and the measured $\mathrm{EC}_{50}$ value is in agreement with what has have been previously reported.

A growing body of evidence suggests that HEK293 cells are a suitable platform for expressing GPCRs. HEK293 cells have successfully been used to produce rhodopsin and the olfactory receptor hOR17-4 ${ }^{13,14,23}$. All of the tested cultures yielded large quantities of receptors that could be easily purified using the rholD4 epitope and monoclonal antibody. Our study further showed that removal of predicted glycosylation sites does not inhibit membrane localization or ligand binding. Although our study only used cells grown on tissue culture plates, previous reports have shown that HEK293 cells can be grown in suspension to yield even larger quantities of receptors ${ }^{23}$.

Because the optimal detergent for a given membrane protein must currently be determined empirically, finding a detergent that can stabilize a class of membrane proteins would be an important contribution. This would save time and resources, and also shorten the path towards studying more membrane proteins. Our results contribute to a growing body of evidence that FC14 may be a promising detergent for stabilizing diverse GPCRs. In addition to hVN1R1, it has solubilized and stabilized various other GPCRs, including several 

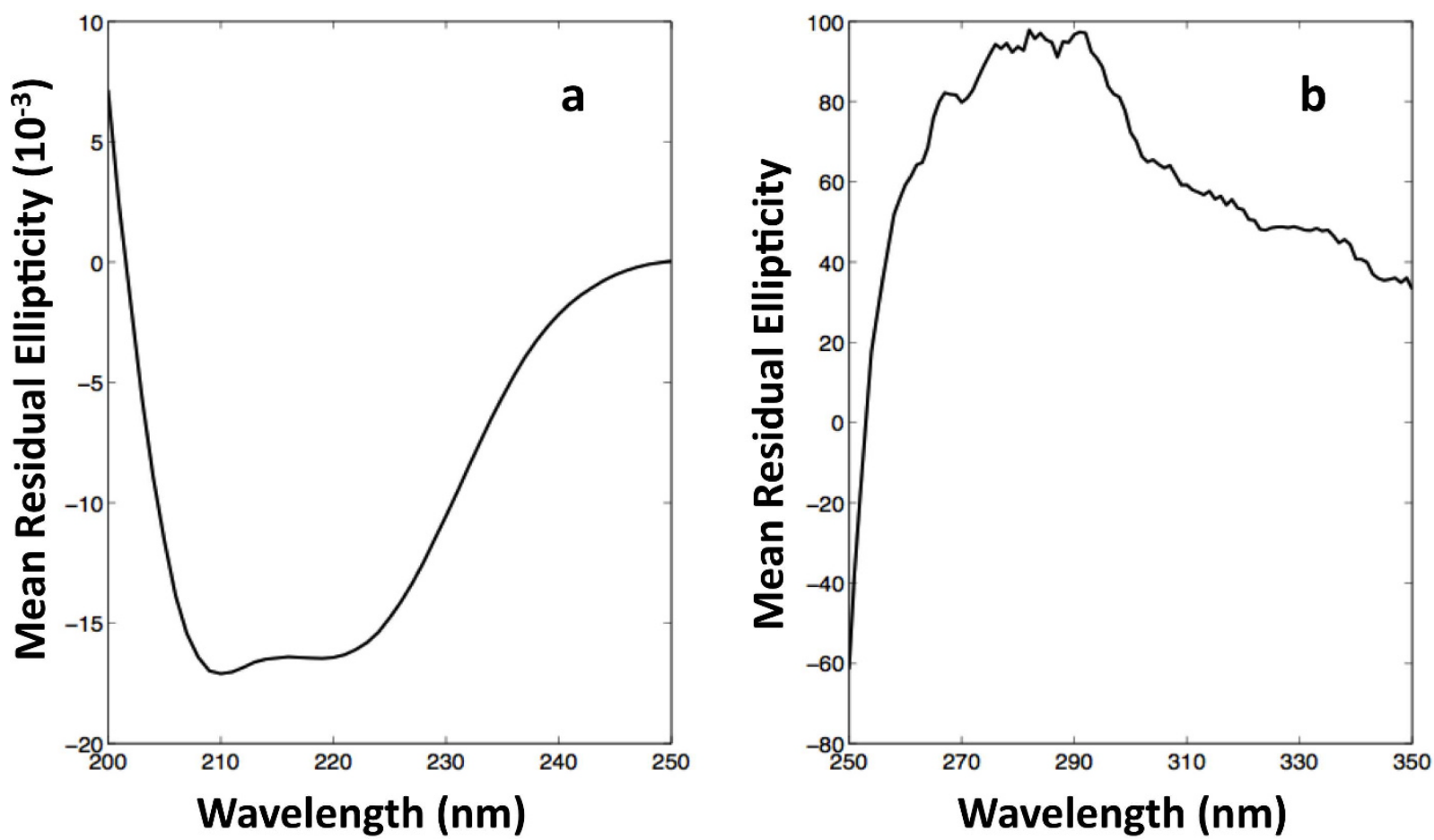

Figure $4 \mid$ Circular dichroism spectra of purified hVN1R1. (a) Far UV trace showing a curve characteristic of an alpha-helix. This shape is expected, as hVN1R1 is a GPCR with seven predicted transmembrane helices. (b) Near UV trace. The peaks indicate that the protein has a well-defined tertiary structure.

chemokine receptors (CCR3, CCR5, CXCR4, CX3CR1), and several olfactory receptors ${ }^{13,22,25}$. Although FC15 and FC16 were able to solubilize a greater quantity of expressed receptor than FC14, all three detergents were able to solubilize comparable amounts. Since
FC14 has a CMC that is high enough to allow for detergent exchange, has been used to obtain high resolution protein structures ${ }^{26,27}$, and can be used for both the solubilization and purification processes, it appears to be a promising choice in membrane protein studies.

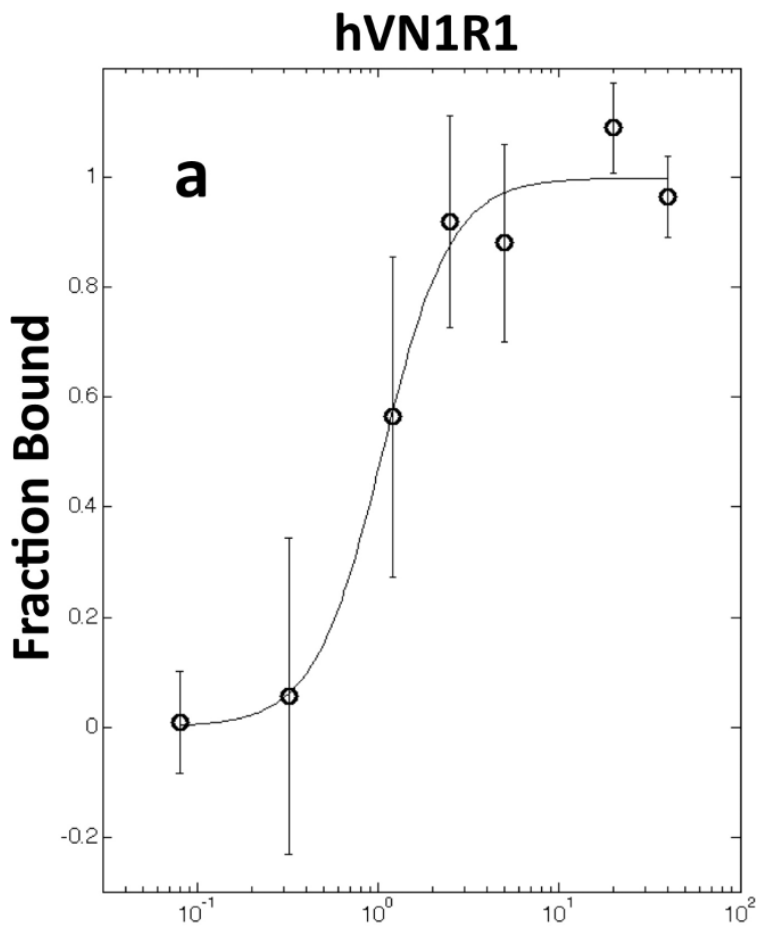

Myrtenal Concentration ( $\mu \mathrm{M})$

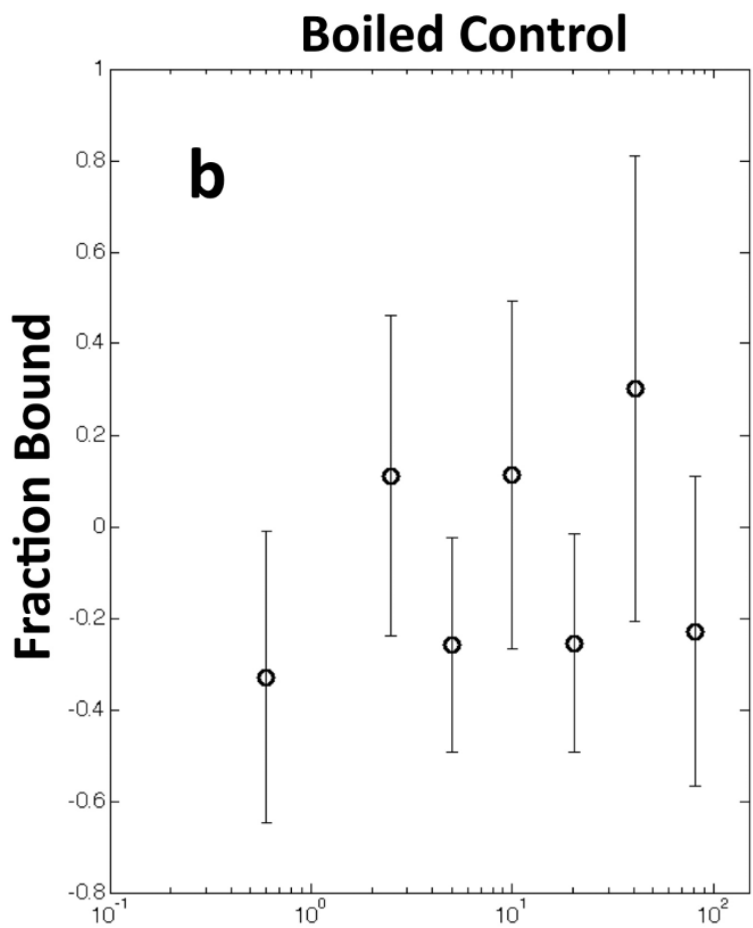

\section{Myrtenal Concentration $(\mu \mathrm{M})$}

Figure 5 | Ligand binding assay of purified hVN1R1 using micro-scale thermophoresis. (a) Samples of hVN1R1 were titrated against varying concentrations of myrtenal, and its thermophoretic mobility was measured. The plateaus at low and high myrtenal concentrations indicate that the receptor is binding its ligand with an $\mathrm{EC} \sim 1 \mu \mathrm{M}$. (b) A boiled control did not exhibit the characteristic sigmoidal binding curve, indicating that the assay was indeed measuring binding between hVN1R1 and myrtenal. Measurements were performed in triplicate. 
Our experimental results suggest that humans have functional VNR genes, while previous reports suggest that human VN1Rs may function more like olfactory receptors. This is the first report showing direct binding between a vomeronasal receptor and a volatile ligand. Our study and previous reports show that they bind volatile molecules with $\mu \mathrm{M}$ binding affinities ${ }^{11}$, they can couple to the canonical OR signaling pathway via G $\alpha$ olf and $G \alpha$ s and $c A M P^{11}$, and hVN1R1 mRNA has been detected in the human MOE ${ }^{12}$. Indeed, mouse VN1Rs signal through TRP, which is a pseudogene in humans. If human VN1Rs are biologically functional, they must have a different signaling mechanism that still needs to be elucidated. The HEK293 cells used in this study provide an excellent mechanism to perform such studies. These cells have been successfully used to express and purify other GPCRs including the olfactory receptor OR17- $4^{13,14,23}$. They can thus be used as a platform to compare OR and VNR function and signaling. Additionally, although the human VNO may not be a functional organ, expression of hVN1R1 mRNA in other tissues in addition to the MOE raises the possibility that it may have non-olfactory functions. Indeed, OR17-4 functions in both olfaction and sperm migration ${ }^{34}$.

Membrane proteins are not only critical for communication between the cellular and external environments, they also have potential uses in medicine and the design of biotechnology-based devices. They comprise nearly $25-30 \%$ of genes in the sequenced genomes, and are involved in various processes ranging from chemical transport, signal transduction, and photosynthesis. GPCRs, the largest subclass of membrane proteins, are the target of nearly $50 \%$ of pharmaceutical drugs. Olfactory-related proteins (including VNRs) are the largest subclass of GPCRs, and could potentially be used to develop highly sensitive detectors.

Detailed structural knowledge of membrane proteins is critical for developing new technologies and medicines. Yet, in spite of their enormous potential, very little is known about membrane protein structures. Of the $\sim 76,000$ protein structures known as of October 2011 , only 302 are unique membrane proteins, and only 6 are unique GPCRs. To facilitate protein structure determination, fast and reliable methods of protein expression and purification must be developed. In addition to hVN1R1, the methods used in this study have been successfully used with bovine rhodopsin and OR17-4. Here, we further show that expressed proteins are functional and targeted to the membrane even when potential glycosylation sites have been removed. This further underscores the potential of this system to be used with a wide variety of membrane proteins in preparation for crystallization trials. Our efforts to purify hVN1R1 may not only help understand the role and function of the human vomeronasal system, but could also contribute toward other membrane protein studies and the development of biologically-inspired technologies like artificial noses.

\section{Methods}

hVN1R1 gene design and construction. The protein sequence for hVN1R1 was obtained from GenBank (AAG10698). To enable expression and purification from mammalian cells, the following modifications were made: 1) addition of a C-terminal rho tag (TETSQVAPA) preceded by a two glycine linker; 2 ) human codon optimization; 3 ) addition of a Kozak sequence $5^{\prime}$ to the start codon; 4) addition of a $5^{\prime}$ EcoRI site and a $3^{\prime}$ XhoI site to facilitate subcloning into expression vectors; 5) addition of an N-terminal strep tag (ASWSHPQFEK) followed by a GSSG linker for further purification; and 6) N117Q, N151Q, N183Q, N198Q, and N256Q mutations to facilitate crystallization. The genes were constructed by Geneart and ligated into the pcDNA 4/To vector (Invitrogen, Carlsbad, CA). The plasmid was amplified in subcloning efficiency DH5a E. Coli (Invitrogen) and purified using Mini- or MaxiPrep kits (Quiagen, Valencia, CA). The transmembrane and loop domains were predicted using the TMHMM Server v 2.0 (http://www.cbs.dtu.dk/services/ TMHMM/). N-linked glycosylation sites were predicted using the NetNGlyc 1.0 Server (http://www.cbs.dtu.dk/services/NetNGlyc/).

Construction of stable inducible hVN1R1 HEK293G cell lines. HEK293S $\mathrm{N}$-acetylglucosaminyltransferase I-negative cells (HEK293G) containing the pcDNA6/Tr vector ${ }^{13}$ were transfected with the pcDNA4/To-hVN1R1 vector using Lipofectamine 2000 (Invitrogen) according to the manufacturer's instructions.
Forty-eight hours after transfection, selective media containing $5 \mu \mathrm{g} / \mathrm{ml}$ of blasticidin and $50 \mu \mathrm{g} / \mathrm{ml}$ zeocin was added. Cells were re-seeded at low density and grown until individual colonies formed. Forty-eight colonies were picked and screened for inducible hVN1R1 expression. Cells were treated with plain media, media supplemented with $1 \mu \mathrm{g} / \mathrm{ml}$ tetracycline, and media supplemented with $1 \mu \mathrm{g} / \mathrm{ml}$ tetracycline and $1,2.5$, or $5 \mathrm{mM}$ sodium butyrate. Two days after induction, cells were scrape-harvested and solubilized in PBS with $2 \%$ w/v Fos-Choline 14 (FC14) (Anatrace) and protease inhibitors (Roche) for $1 \mathrm{hr}$ at $4^{\circ} \mathrm{C}$. Cell lysates were centrifuged for 30 minutes at 10,000 rpm to remove insoluble debris. Dot blots and Western-blots were used to compare protein expression among clones. The clone with the highest expression when induced, the least detectable expression when not induced, and least toxicity upon induction, was selected and expanded for future experiments. All cultures were grown in DMEM F12 with GlutaMAX (Invitrogen) supplemented with $10 \%$ fetal bovine serum (Invitrogen), $15 \mathrm{mM}$ HEPES (Invitrogen), $0.1 \mathrm{mM}$ non-essential amino acids (Invitrogen), $0.5 \mathrm{mM}$ sodium pyruvate (Invitrogen), $100 \mathrm{Units} / \mathrm{ml}$ penicillin and $100 \mu \mathrm{g} / \mathrm{ml}$ streptomycin (Invitrogen). The expanded stable hVN1R1 clone was maintained in media that also contained $5 \mu \mathrm{g} / \mathrm{ml}$ blasticidin and $25 \mu \mathrm{g} / \mathrm{ml}$ zeocin. All cells were grown at $37^{\circ} \mathrm{C}$, $5 \% \mathrm{CO}_{2}$, and $95 \%$ relative humidity.

Immunocytochemistry. Vomeronasal receptors were visualized using a rho1D4 primary antibody. Cells were seeded at low density on poly-L-lysine (Sigma-Aldrich) coated glass coverslips. After one day, cells were induced with $1 \mu \mathrm{g} / \mathrm{ml}$ tetracycline and $1 \mathrm{mM}$ sodium butyrate. One day after induction, the media was removed. Cells were gently washed with PBS and fixed for 20 minutes in $10 \%$ neutral buffered formalin (Sigma-Aldrich) at room temperature. Permeabilized $(1: 1$ acetone:methanol, 3 minutes, $-20^{\circ} \mathrm{C}$ ) and non-permeabilized cells were then blocked in PBST (PBS, 0.2\% tween-20, $0.3 \mathrm{M}$ glycine, 4\% serum) for 1 hour at room temperature, and incubated with the primary antibody solution ( $1: 1500$, PBS, $0.2 \%$ tween-20, $4 \%$ serum) overnight at $4{ }^{\circ} \mathrm{C}$. The labeled protein was visualized with Alexaflour-488 goat-anti-mouse secondary antibody conjugate $(1: 3000$, PBS, 1 hour, room temperature). Slides were mounted using ProLong Gold Antifade with DAPI.

Cell extract preparation. Cells were grown on plates as previously described ${ }^{13}$. When the appropriate density was reached, cells were induced with $1 \mu \mathrm{g} / \mathrm{ml}$ tetracycline and $1 \mathrm{mM}$ sodium butyrate. Two days after induction, cells were scrape harvested, pooled, snap frozen in liquid nitrogen, and stored at $-80^{\circ} \mathrm{C}$ until used for future experiments.

Detergent Screening. Frozen cell pellets were thawed on ice and resuspended in PBS containing protease inhibitors (Roche). Detergents were added to a final concentration of $2 \% \mathrm{w} / \mathrm{v}$. The suspensions were rotated for 1 hour at $4{ }^{\circ} \mathrm{C}$ to solubilize the protein, and were spun at 13,000 rpm for 30 minutes to remove insoluble fractions. Relative protein solubilization in each detergent was assayed with a dot blot. Ninety-six detergents were selected for screening as previously described ${ }^{22}$.

Receptor purification. Rho1D4 immunoaffinity purification has been previously described $^{13,23}$. Briefly, frozen cell pellets were thawed on ice. Cells were resuspended in PBS containing protease inhibitors. PBS containing FC-14 was added to a final concentration of $2 \% \mathrm{w} / \mathrm{v}$ FC-14. The final liquid:cell ratio was $12.5 \mathrm{ml} / 1 \mathrm{~g}$ cells. The protein was solubilized by rotating for 4 hours at $4{ }^{\circ} \mathrm{C}$. The non-solubilized fraction was pelleted by centrifuging for 30 minutes at $30,000 \mathrm{~g}$ at $4^{\circ} \mathrm{C}$. The solubilized fraction was incubated with DNAse $(1: 2000)$ and RNAse $(1: 1000)$ for 15 minutes on ice. Rho1D4-coupled CNBr-activated Sepharose 4B beads (GE Healthcare) were added to the cell extract supernatant (binding capacity $0.7 \mathrm{mg} / \mathrm{ml}$ ); receptors were captured by rotating the mixture overnight at $4^{\circ} \mathrm{C}$. The beads were collected by centrifuging at $1400 \mathrm{rpm}$ for 1 minute, or filtering the supernatant through a filter column (Biorad). The supernatant was saved for future analysis and labeled as "flow through". The beads were resuspended in 1 bead volume of wash buffer (PBS $+0.2 \%$ w/v FC-14), rotated for 10 minutes at $4{ }^{\circ} \mathrm{C}$, and re-pelleted. Washes were performed until the total protein concentration in the washes was less than $0.01 \mathrm{mg} / \mathrm{ml}$ (NanoDrop). One bead volume of elution buffer (PBS $+0.2 \%$ w/v FC-14 $+200 \mathrm{uM}$ Ac-TETSQVAPA- $\mathrm{NH}_{2}$ peptide) was then added to the beads. Elutions were performed until the total protein concentration was less than $0.01 \mathrm{mg} / \mathrm{ml}$.

Size exclusion chromatography was used to separate the monomeric and higher molecular-weight forms of the receptor. A Hi-Load 16/60 Supradex 200 column with an Akta Purifier HPLC system (GE Healthcare) was used. The column was first equilibrated with at least 1 column volume of wash buffer. Protein samples were concentrated to 1.5-3 ml using a 50,000 MWCO filter column (Millipore), loaded on the column, and run with wash buffer at $0.3 \mathrm{ml} / \mathrm{min}$. Fractions exiting the column were automatically collected; protein content was monitored with UV absorbance at $215 \mathrm{~nm}, 254 \mathrm{~nm}$, and $280 \mathrm{~nm}$. Peak fractions were pooled, concentrated, and analyzed with Western blotting and silver staining (SilverXpress, Invitrogen). A calibration to correlate the column exit time with the molecular mass was previously performed in our lab, and is described in [23].

GPCR Detection and Purity Analysis. Western blots and silver stains were used to detect the proteins and analyze their purity. Samples were prepared and loaded in Novex 10\% Bis-Tris SDS-PAGE gels (Invitrogen) according to the manufacturer's protocol, with the exception that the samples were incubated at room temperature prior to loading as boiling causes membrane protein aggregation. For blotting, the gel-resolved samples were transferred to a nitrocellulose membrane, blocked in milk $(5 \% \mathrm{w} / \mathrm{v}$ non-fat dried milk in TBST) for 1 hour, and incubated with a rholD4 
primary antibody ( $1: 3000$ in TBST, 1 hour at room temperature, or overnight at $4^{\circ} \mathrm{C}$ ). The GPCRs were then detected with a goat anti-mouse HRP-conjugated secondary antibody (Pierce, Rockford, IL) $(1: 5000$ in TBST, 1 hour, room temperature) and visualized using the ECL-Plus Kit (GE Healthcare). The SilverXpress kit (Invitrogen, LC6100) was used according to the manufacturer's instructions to perform total protein stains of the samples. All images were captured using a Fluor Chem gel documentaion system (Alpha Innotech, San Leandro, CA). ImageJ software $[27,28]$ was used to compare band intensities and analyze sample purity.

Circular Dichroism Spectroscopy. Far UV CD spectra were measured over the wavelengths $200 \mathrm{~nm}-260 \mathrm{~nm}$ with a CD spectrometer (AVIV Biomedical Model 202). Measurements were made at $15^{\circ} \mathrm{C}$, with a step size of $1 \mathrm{~nm}$ and an averaging time of 4 seconds. Measurements for each sample were made in triplicate and averaged. Protein samples were concentrated to at least $0.1 \mathrm{mg} / \mathrm{ml}$ for far UV readings, and $1.7 \mathrm{mg} / \mathrm{ml}$ for near UV readings; concentrations were determined by averaging ten spectroscopic measurements (Nanodrop). The protein spectra were blanked to the spectrum obtained for wash buffer. A QS quartz cuvette (Hellma) with a $1 \mathrm{~mm}$ path length was used to perform all experiments.

Microscale Thermophoresis Ligand Binding Assay. Thermophoresis was used to measure the binding interactions between purified receptors and their ligands using a setup similar to that previously described ${ }^{30,31}$. To eliminate artifacts caused by labeling or modifying proteins, the fluorescence of native GPCR tryptophans was used to monitor the local receptor concentration. For each tested GPCR, a titration series with constant receptor concentration and varying ligand concentrations was prepared in a final solution of $10 \%$ DMSO and $0.2 \%$ FC-14 in PBS. Potential autofluorescence of each ligand was checked: no fluorescence signal was detected from the ligands in the tryptophan fluorescence channel. The final receptor concentration was $2 \mu \mathrm{M}$. Approximately $1.5 \mu \mathrm{l}$ of each sample was loaded in a fused silica capillary (Polymicro Technologies, Phoenix, USA) with an inner diameter of $300 \mu \mathrm{m}$. An infrared laser diode was used to create a $0.12 \mathrm{~K} / \mu \mathrm{M}$ temperature gradient inside the capillaries (Furukawa FOL1405-RTV-617-1480, wavelength $\lambda=1480 \mathrm{~nm}$, $320 \mathrm{~mW}$ maximum power, AMS Technologies AG, Münich Germany). Tryptophan fluorescence was excited with a UV-LED $(285 \mathrm{~nm})$, and was measured with a $40 \mathrm{x}$ SUPRASIL synthetic quartz substrate microscope objective, numerical aperture 0.8 (Partec, Goerlitz, Germany). The local receptor concentration in response to the temperature gradient was detected with a photon counter PMT P10PC (Electron Tubes Inc, Rockaway, NJ, USA). All measurements were performed at room temperature. Fluorescence filters for tryptophan (F36-300) were purchased from AHF-Analysentechnik (Tübingen, Germany).

1. Trotier, D. et al. The vomeronasal cavity in adult humans. Chem. Senses $\mathbf{2 5}$, 369-380 (2000).

2. Bossy, J. Development of olfactory and related structures in staged human embryos. Anat. Embryol. 161, 225-236 (1980).

3. Boehm, N., Roos, J., Gasser, B. Luteinizing hormone-releasing hormone (LHRH)expressing cells in the nasal septum of human fetuses. Dev. Brain Res. 82, 175-180 (1994).

4. Humphrey, T. The development of the olfactory and the accessory olfactory formations in human embryos and fetuses. J. Comp. Neurol. 73, 431-468 (1940).

5. Meisami, E., and Meisami, E., and Bhatnagar, K. P. Structure and diversity in mammalian accessory olfactory bulb. Microscopy Research and Technique 43, 476-499 (1998).

6. Boehm, N., and Gasser, B. Sensory receptor-like cells in the human foetal vomeronasal organ. NeuroReport 4, 867-870 (1993).

7. Takami, S. et al. Vomeronasal epithelial cells of the adult human express neuronspecific molecules. Neuroreport 4, 375-378 (1993).

8. Grosser, B. I., Monti-Bloch, L., Jennings-White, C., Berliner, D. L. Behavorial and electrophysiological effects of androstadienone, a human pheromone. Psychoneuroendocrino 25, 289-99 (2000).

9. Berliner, D. L., Monti-Bloch, L., Jennings-White, C., Diaz-Sanchez, V. The functionality of the human vomeronasal organ (VNO): Evidence for steroid receptors. J. Steroid Biochem. Molec. Biol. 58, 259-265 (1996).

10. Rodriguez, I., and Mombaerts, P. Novel human vomeronasal receptor-like genes reveal species-specific families. Curr. Biol. 12, R409-411 (2002).

11. Shirokova, E., Raguse, J. D., Meyerhof, W., Krautwurst, D. The human vomeronasal type-1 receptor family - detection of volatiles and cAMP signaling in HeLa/Olf cells. FASEB J. 22, 1416-1425 (2008).

12. Rodriguez, I., Greer, C. A., Mok, M. Y., Mombaerts, P. A putative pheromone receptor gene expressed in human olfactory mucosa. Nat. Genet. 26, 18-19. (2000).

13. Cook, B., Ernberg, K. E., Chung, H., Zhang, S. Study of a synthetic human olfactory receptor 17-4: Expression and purification from an inducible mammalian cell line. PLoS One 3 (8): e2920. doi: 10.1371/journal.pone.0002920. (2008).

14. Reeves, P. J., Thurmond, R. L., Khorana, H. G. Structure and function in rhodopsin: high level expression of a sythetic bovine opsin gene and its mutants in stable mammalian cell lines. Proc. Natl. Acad. Sci. USA 93, 11487-11492 (1996).
15. Gat, U., Nekrasova, E., Lancet, D., Natochin, M. Olfactory receptor proteins. Expression, characterization and partial purification. Eur. J. Biochem. 225, 11571168 (1994).

16. Nekrasova, E., Sosinskaya, A., Natochim, M., Lancet, D., Gat, U. Overexpression, solubilization and purification of rat and human olfactory receptors. Eur. J. Biochem. 238, 28-37 (1996).

17. Katada, S., Tanaka, M., Touhara, K. Structural determinants for membrane trafficking and G protein selectivity of a mouse olfactory receptor. J. Neurochem. 90(6), 1453-1463 (2004).

18. Gimelbrant, A. A., Haley, S. L., McClintock, T. S. Olfactory receptor trafficking involves conserved regulatory steps. J. Biol Chem. 276(10), 7285-7290 (2001)

19. Lu, M., Staszewski, L., Escheverri, F., Xu, H., Moyer, B. D. Endoplasmic reticulum degradation impedes olfactory G-protein coupled receptor functional expression. BMC Cell Biol. 5,34 (2004).

20. Lu, M., Echeverri, F., Moyer, B. D. Endoplasmic reticulum retention, degradation, and aggregation of olfactory G-protein coupled receptors. Traffic 4, 416-433 (2003).

21. Jayadev, S., Smith, R. D., Jagadeesh, G., Baukal, A. J., Hunyady, L. N-linked glycosylation is required for optimal AT1a angiotensin receptor expression in COS-7 cells. Endocrinology 140, 2010-2017 (1999).

22. Ren, H., Yu, D., Ge, B., Cook, B., Xu, Z., Zhang, S. High-level production, solubilization and purification of synthetic human GPCR chemokine receptors CCR5, CCR3, CXCR4 and CX3CR1. PLoS One 4(2), e4509 doi: 10.1371/journal/ pone.0004509 (2009).

23. Cook, B. et al. Large-scale production and study of a synthetic $\mathrm{G}$ protein-coupled receptor: Human olfactory receptor 17-4. Proc. Natl. Acad. Sci. USA 106(29), 11925-11930 (2009).

24. Gorzelle, B. M., et al. Reconstitutive refolding of diacylglycerol kinase, an integral membrane protein. Biochemistry 38, 16373-16382 (2002).

25. Leck, K-J., Zhang, S., Hauser, C. A. E. Study of bioengineered zebra fish olfactory receptor 131-2: receptor purification and secondary structure analysis. PLOS ONE 5, e15027 (2010).

26. Bass, R. B., Strop, P., Barclay, M., Rees, D. C. Crystal structure of Escherichia coli MscS, a voltage-modulated and mechanosensitive channel. Science 298, 15821587 (2002).

27. Wang, W., et al. The structure of an open form of an E. coli mechanosensitive channel at 3.45 A resolution. Science 321, 1179-1183 (2008).

28. Liu, X., Garriga, P., Khorana, H. G. Structure and function in rhodopsin: Correct folding and misfolding in two point mutants in the intradiscal domain of rhodopsin identified in retinitis pigmentosa. Proc. Natl. Acad. Sci. USA 93, 4554-4559 (1996).

29. Duhr, S. \& Braun, D. Why molecules move along a temperature gradient. Proc Natl Acad Sci USA 103, 19678-19682 (2006).

30. Baaske, P., Wienken, C. J., Reineck, P., Duhr, S., \& Braun, D. Optical thermophoresis for quantifying the buffer dependence of aptamer binding. Angew Chem Int Ed 49, 2238-2241 (2010).

31. Wienken, C. J., Baaske, P., Rothbauer, U., Braun, D., Duhr, S. Protein-binding assays in biological liquids using microscale thermophoresis. Nature Comm. 1, 100 (2010)

32. Pollack, S. J., et al. A comparative study of fragment screening methods on the p38 $\alpha$ kinase: new methods, new insights. J Comput Aided Mol Des. doi: 10.1007/ s10822-011-9454-9 (2011).

33. Kaiser, L., et al. Large-scale production and study of a synthetic $G$ protein-coupled receptor: human olfactory receptor 17-4. Proc Natl Acad Sci USA 105, 1572615731 (2008)

34. Spehr, M., et al. Identification of a testicular odorant receptor mediating human sperm chemotaxis. Science 299, 2054-2058 (2003).

\section{Acknowledgement}

This work is supported in part from Defense Advanced Research Program Agency-HR0011-09-C-0012.

\section{Author contributions}

$\mathrm{KC}, \mathrm{PB}, \mathrm{SG}$, and SZ designed the experiments. KC, PB, SG performed the experiments. KC $\mathrm{PB}, \mathrm{CW}$, and $\mathrm{SZ}$ wrote the manuscript.

\section{Additional information}

Competing financial interests: KC, C.J.W, D.B. and SZ declare no competing financial interests. P.B., S.G., and S.D. are employees of NanoTemper that produces and markets the Microscale Thermophoresis instrument. Publication of this paper may increase others' awareness of the Microscale Thermophoresis instrument and benefit NanoTemper.

License: This work is licensed under a Creative Commons

Attribution-NonCommercial-ShareAlike 3.0 Unported License. To view a copy of this license, visit http://creativecommons.org/licenses/by-nc-sa/3.0/

How to cite this article: Corin, K. et al. Structure and function analyses of the purified GPCR human vomeronasal type 1 receptor 1. Sci. Rep. 1, 172; DOI:10.1038/srep00172 (2011). 